\title{
A combined vacuum crushing and sieving (CVCS) system designed to determine noble gas paleotemperatures from stalagmite samples
}

\author{
Nadia Vogel \\ Department of Water Resources and Drinking Water, Eawag, Swiss Federal Institute of Aquatic Science and \\ Technology, Uberlandstrasse, Dübendorf, Switzerland \\ Department of Earth Sciences, ETH Zurich, Institute of Geochemistry and Petrology, Clausiusstrasse, Zurich, \\ Switzerland(nadia.vogel@eawag.ch)
}

\section{Matthias S. Brennwald}

Department of Water Resources and Drinking Water, Eawag, Swiss Federal Institute of Aquatic Science and Technology, Überlandstrasse, Dübendorf, Switzerland

\section{Dominik Fleitmann}

Department of Earth Sciences, Institute of Geological Sciences, University of Bern, Baltzerstrasse, Bern, Switzerland

Oeschger Center for Climate Research, University of Bern, Baltzerstrasse, Bern, Switzerland

Now at Department of Archaeology, School of Human and Environmental Sciences, University of Reading, Whiteknights, Reading, UK

\section{Rainer Wieler, Colin Maden, and Andreas Süsli}

Department of Earth Sciences, ETH Zurich, Institute of Geochemistry and Petrology, Clausiusstrasse, Zurich, Switzerland

\section{Rolf Kipfer}

Department of Earth Sciences, ETH Zurich, Institute of Geochemistry and Petrology, Clausiusstrasse, Zurich, Switzerland

Department of Environmental Systems Science, ETH Zurich, Institute of Biogeochemistry and Pollutant Dynamics, Universitätsstrasse, Zurich, Switzerland

Department of Water Resources and Drinking Water, Eawag, Swiss Federal Institute of Aquatic Science and Technology, Dübendorf, Switzerland

[1] This paper presents a novel extraction device for water and noble gases from speleothem samples for noble gas paleotemperature determination. The "combined vacuum crushing and sieving (CVCS) system" was designed to reduce the atmospheric noble gas contents from air inclusions in speleothem samples by up to 2 orders of magnitude without adsorbing atmospheric noble gases onto the freshly produced grain surfaces, a process that had often hampered noble gas temperature (NGT) determination in the past. We also present the results from first performance tests of the CVCS system processing stalagmite samples grown at a known temperature. This temperature is reliably reproduced by the NGTs derived from Ar, $\mathrm{Kr}$, and Xe extracted from the samples. The CVCS system is, therefore, suitable for routine determinations of accurate NGTs. In combination with stalagmite dating, these NGTs will allow reconstructing past regional 
temperature evolutions, and also support the interpretation of the often complex stable isotope records preserved in the stalagmites' calcite.

Components: 7,401 words, 5 figures, 2 tables.

Keywords: noble gases; speleothem; stalagmite; temperature determination; paleoclimate.

Index Terms: 1616 Climate variability: Global Change; 1637 Regional climate change: Global Change; 1694 Instruments and techniques: Global Change.

Received 17 January 2013; Revised 30 April 2013; Accepted 30 April 2013; Published 30 July 2013.

Vogel, N., M. S. Brennwald, D. Fleitmann, R. Wieler, C. Maden, A. Süsli, and R. Kipfer (2013), A combined vacuum crushing and sieving system designed to determine noble gas paleotemperatures from stalagmite samples, Geochem. Geophys. Geosyst., 14, 2432-2444, doi:10.1002/ggge.20164.

\section{Introduction}

[2] Stalagmites have been recognized as exceptionally valuable paleoclimate archives, as they occur widespread in continental settings, cover time intervals of up to $10^{5} \mathrm{a}$, and are precisely and accurately dateable. Their stable isotope records allow us to retrieve a wealth of high-resolution paleoclimate information [e.g., Fairchild and Baker, 2012]. However, a fundamental piece of paleoclimate information has proven difficult to be deduced from speleothem samples, that is the cave temperature at which the calcite of the growing speleothem precipitated. As the cave temperature is very similar to the mean annual temperature above the cave [e.g., Fairchild et al., 2006], such local temperature histories provide important information about the respective regional paleotemperature evolution. First attempts to estimate temperatures were made using the oxygen isotopic composition of the stalagmites' calcite [e.g., Hendy and Wilson, 1968; Thompson et al., 1974], which, however, turned out to be notoriously complex in its interpretation. In the past few years, other methods have been developed, including for example clumped isotope thermometry [e.g., Affek et al., 2008], liquid-vapor homogenization of stalagmite fluid inclusions [Krüger et al., 2011], or $\mathrm{D} / \mathrm{H}$ ratios of stalagmite water [e.g., Zhang et al., 2008]. Two research groups have also explored the possibility to deduce temperature information from the concentrations of noble gases dissolved in the inclusion water of stalagmites [Kluge et al., 2008; Scheidegger et al., 2010; Scheidegger et al., 2011]. This technique of "noble gas temperature (NGT) determination" is being routinely applied to groundwaters and lakes, and is based on the temperature-, pressure-, and salinity-dependent solubilities of atmospheric gases in water [e.g., Brennwald et al., 2013; Kipfer et al., 2002]. Application of the noble gas thermometer to stalagmite water is analytically challenging, as the amounts of both water and dissolved noble gases are very small and therefore difficult to analyze. An additional complication emerges from the fact that stalagmites do not only contain water-filled inclusions but also inclusions filled with air [Scheidegger et al., 2010; Scheidegger et al., 2011]. The noble gas content of an air-filled inclusion is orders of magnitude higher than that of a water-filled inclusion of comparable size. If noble gases are extracted from a stalagmite sample by "simple" total crushing or heating techniques, the air and water contents of both types of inclusions are extracted together, and noble gases from air-filled inclusions often overwhelm the amounts of noble gases dissolved in the inclusion water [e.g., Kluge et al., 2008; Scheidegger et al., 2010]. In such cases, a reliable deconvolution of both components becomes impossible, prohibiting a NGT determination from the amounts of noble gases originally dissolved in the inclusion water [Kluge et al., 2008; Scheidegger et al., 2010]. Attempts to reduce the air content of a sample by crushing it to a predefined grain size prior to water extraction indeed reduced the atmospheric noble gas fraction in the crushed sample by about 1-2 orders of magnitude [Scheidegger et al., 2011]. However, this offline crushing had to be performed in air or a helium atmosphere, as crushing a sample to a predefined grain size in vacuum was not possible at that time. The procedure, therefore, often introduced artefacts, mainly by adding an illdefined adsorbed atmospheric noble gas component to the sample gas, which again often prevented NGT determination [Scheidegger et al., 2010, 2011]. For a detailed discussion of the results 
obtained by Scheidegger et al. [2010, 2011] from offline crushing experiments on stalagmites with a special focus on biases introduced during sample handling, see, Brennwald et al. [2013].

[3] In order to overcome these experimental limitations, we have developed a water and noble gas extraction system that clears the way for routine determination of robust NGTs from stalagmite (and other fluid inclusion bearing) samples. This paper describes the design and operation of the new "combined vacuum crushing and sieving (CVCS) system", and also presents results from first performance tests of the CVCS system, for which samples of a stalagmite were processed, whose calcite precipitated at a known temperature.

[4] We show that the new CVCS technique can provide local temperature estimates with overall uncertainties of $\sim 1-2^{\circ} \mathrm{C}(1 \sigma)$. The resulting NGTs will thus contribute to a better understanding of the temperature evolution of the region above the respective host cave. In addition, NGTs will help interpreting the often complex stable isotope records preserved in stalagmites, because noble gas dissolution in stalagmite water is, except for the temperature, independent of most of the other climate variables that influence the stable isotope composition of the precipitating calcite like, e.g., the sources and rates of rainfall, or drip water evaporation from the active surface of the growing stalagmite [e.g., Fairchild and Baker, 2012].

\section{A New Water and Noble Gas Extraction System for Stalagmite Samples}

[5] The key to a successful NGT determination is to crush a stalagmite sample to a grain size larger than the typical dimensions of water-filled inclusions, but small enough to break open the usually somewhat larger air-filled inclusions prior to water and noble gas extraction [cf., Scheidegger et al., 2010]. Crushing considerably reduces the air/ water volume ratio of the respective sample [Scheidegger et al., 2010, 2011]. Identifying this "ideal" grain size obviously requires a microscopic investigation of the stalagmite samples prior to NGT determination.

[6] Previous experiments have also shown that crushing has to be performed in vacuum, in order to avoid adsorption of atmospheric noble gases to the sample, as this process fractionates elementally the atmospheric noble gas composition. We therefore developed an extraction device that integrates both of these prerequisites, i.e., which allows in vacuo crushing of a sample to a continuously adjustable maximum grain size and a further separation of the desired (medium) grain size fraction from larger and smaller grains by in vacuo sieving of the crushed sample. While we expect that the medium grain-size fraction yields the lowest air/ water volume ratio and, hence, is most suitable for accurate NGT determination, all three grain size fractions resulting from the crushing procedure are available for analysis.

[7] Figure 1 shows a schematic drawing and a photograph with the most important components of the CVCS system: the crushing chamber with the crushing table and piston, the sieving unit, and the three glass fingers to collect the coarse, medium, and fine grain size fractions, from which water and noble gases are extracted by heating. In the following, we describe the design and operation of the new CVCS system.

\subsection{Loading of Samples and Evacuation of the CVCS System}

[8] One sample with a total mass of up to $\sim 2 \mathrm{~g}$ can be loaded at a time. The sample is ideally split into several cubes of up to $\sim 5 \mathrm{~mm}$ size. Up to four cubes can be loaded into a manually operated in vacuo sample feeder located at the back of the crushing chamber (not visible in Figure 1), and an additional fifth cube can be placed directly onto the crushing table. Also the two sieves, whose mesh sizes are selected according to the desired grain sizes of the three future grain size fractions, have to be mounted into the sieve holders prior to closing the crusher chamber. The latter is done by mounting the front plate of the crusher chamber and sealing it by a Viton ${ }^{\circledR}$ gasket. The system is then pumped to a pressure of $\sim 10^{-6}$ mbar. The partial pressure of the remaining atmospheric noble gases in the crusher chamber is several orders of magnitude lower than, e.g., in the helium-filled glove box used for offline crushing by Scheidegger et al. [2010, 2011]. The system is pumped for at least $12 \mathrm{~h}$ before the sample is crushed.

\subsection{Vacuum Crushing and Sieving}

[9] Crushing of a sample cube is performed by applying controlled force via a piston pressed onto the cube by manually turning the top handle (Figure 1). Simultaneously turning the front handle (Figure 1) also leads to a small eccentric rotation of the piston, which is crucial to produce a loose granular powder instead of an undesired pressed pellet of stalagmite material. The final distance 


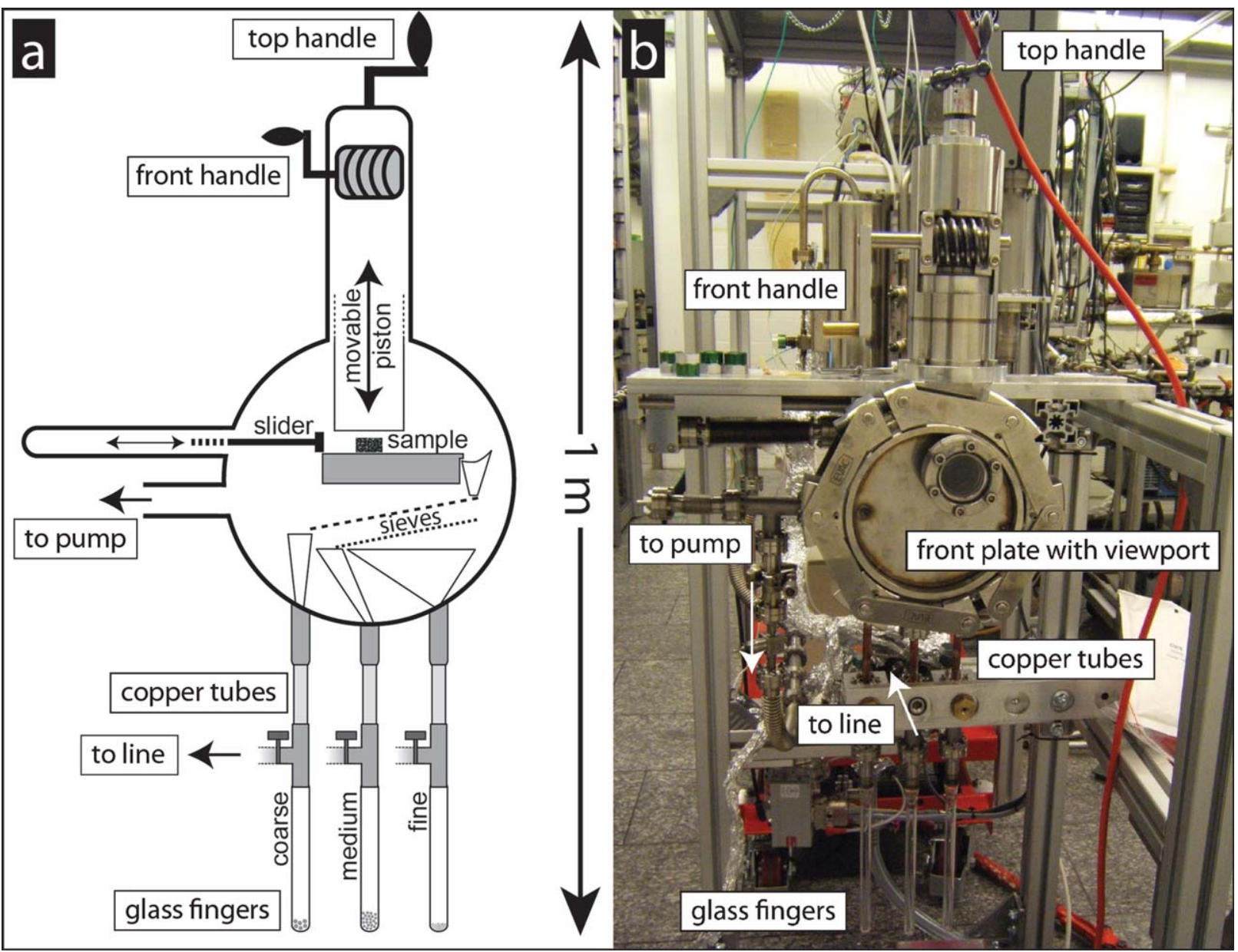

Figure 1. (a) Schematic drawing and (b) photograph of the CVCS system. The overall vertical extension of the crusher with sample fingers is $\sim 1 \mathrm{~m}$. Not visible is the sample feeder on the back of the crushing chamber, from which calcite cubes are transported one by one to the sample table for crushing.

between piston and crushing table determines the approximate maximum grain size of the crushed material and can be set with a resolution of $\sim 10$ $\mu \mathrm{m}$. The crushing process can be observed through a glass viewport in the front plate of the crusher chamber. During the entire crushing and subsequent sieving procedure the CVCS system remains permanently pumped. Air released from the sample is, therefore, instantly removed, avoiding adsorption of the indigenous air-related noble gases onto the freshly produced grain surfaces. After the first sample cube has been crushed, the piston is lifted. Without breaking the vacuum, the sample powder is now transferred to a set of two sieves using a slider, which simultaneously strips off the powder from the crushing table and also powder sticking to the bottom of the piston. Depending on the mesh sizes of the two sieves, the crushed material is separated into a coarse, a medium, and a fine grain size fraction, each of which is funneled into its respective glass finger. To support sieving, the sieve holder is gently tapped in vacuo via a metal pole. Once the first batch of powder is transferred to the glass fingers, the next sample cube is transported to the crushing table while the system remains evacuated, and the crushing and sieving procedure is repeated. Processing the 4-5 sample cubes requires $\sim 30-45 \mathrm{~min}$.

\subsection{Extraction of Water and Noble Gases from the Grain Size Separates}

[10] After the crushing and sieving procedure is terminated, the three filled glass fingers are separated from the crusher chamber to enable individual processing of each grain size fraction. This is done by sealing the copper tube connections (Figure 1) between the glass fingers and the crusher chamber with a crimping tool. The glass fingers are then pumped for at least another $24 \mathrm{~h}$ via the valves connecting them to the extraction line 
(Figure 1) to minimize residual adsorbed water and noble gases released during crushing of the sample. Subsequently, water and noble gases are extracted from a first grain size fraction by heating the respective glass finger. Heating to between 200 and $300^{\circ} \mathrm{C}$ for $1 \mathrm{~h}$ usually opens enough waterfilled inclusions for water and noble gas analysis. At higher temperatures considerably more inclusions could be extracted [Vogel et al., 2013], but at the expense of also producing considerable amounts of reactive gases, which are not fully removed in our purification line and would interfere with the noble gas measurements.

\subsection{Preparation of the Crusher for a New Sample}

[11] Once the three grain size fractions have been analyzed, crucial parts of the CVCS system can be conveniently removed for cleaning before a new sample is loaded. Also, the sealed copper tubes connecting the crusher chamber with the glass fingers have to be replaced. After new sample cubes have been loaded and the front plate has been reattached, the CVCS system is evacuated again and the empty glass fingers are baked at $350^{\circ} \mathrm{C}$ for several hours before a new sample is crushed.

\subsection{Samples}

[12] The performance of the CVCS system was tested on samples of stalagmite D1 from Dimarshim Cave, Socotra Island (Yemen), which already was studied by Scheidegger et al. [2010, 2011]. Our new data can, therefore, be directly compared with the results from previous extraction techniques. Stalagmite D1 is also well dated [Fleitmann et al., 2007] and, most importantly, the cave temperature, at which it grew in the past, is well constrained. Samples used for this study grew around 400 a BP (samples D1.1 and D1.2) and 2400 a BP (sample D1), respectively, and at temperatures between 25 and $27^{\circ} \mathrm{C}$, i.e., very similar to the modern mean annual air temperature in Dimarshim Cave of $\sim 27^{\circ} \mathrm{C}$. This temperature constraint is based, e.g., on sea surface temperature (SST) reconstructions in the Arabian Sea. Doose-Rolinski et al. [2001] report SSTs between 25 and $27.5^{\circ} \mathrm{C}$ during the past 5000 years and a rather stable SST over the last 2000 a BP of $\sim 26.5^{\circ} \mathrm{C}$ in the northeastern Arabian Sea. Similarly, SST reconstructions from sediment cores taken in the western Arabian Sea indicate a maximum increase of the SST of $2^{\circ} \mathrm{C}$ over the past $23 \mathrm{ka}$ toward the present day annual mean SST of $\sim 26^{\circ} \mathrm{C}$ [Huguet et al., 2006].

[13] The three samples processed for the tests had total masses between 1.4 and $2 \mathrm{~g}$ (corresponding to a temporal resolution of several tens of years based on an average growth rate of the selected stalagmite of $\sim 0.25 \mathrm{~mm} \mathrm{a}^{-1}$ ) [cf., Vogel et al., 2013], and were each cut into four to five cubes. The most suitable grain size for NGT determination had been determined by microscopic observation to be 250-350 $\mu \mathrm{m}$ [Scheidegger et al., 2010]. Accordingly, the final distance between piston and crushing table was set to $350 \mu \mathrm{m}$ and the selected sieve mesh sizes were 400 and 200 $\mu \mathrm{m}$, respectively. Note that grains larger than 400 $\mu \mathrm{m}$ do occur because calcite usually breaks into elongate grains. With this setup, the grains with sizes of 250-350 $\mu \mathrm{m}$ and, presumably, the most suitable, i.e., the lowest, air/water volume ratio, are expected in the medium grain size fraction. For each sample, the distribution of the mass of sample material into the three grain size fractions is documented in Table 1 (column 2). By selecting different setups (i.e., different final distances between piston and crushing table, different sieve mesh sizes), the system can be optimized to process samples with very different requirements for grain sizes. Sample recovery, i.e., the cumulative mass of the three grain size fractions divided by the mass of the original sample, varies between $\sim 0.6$ and 0.8. Sample material is lost during the transfer of sample powder from the crushing table to the funnel and from the sieves to the sample fingers.

\section{Water and Noble Gas Measurements}

\subsection{Water Vapor Pressure Measurement and Gas Purification for Noble Gas Analysis}

[14] The mass of water extracted from a grain size fraction is determined by cryogenically concentrating the water vapor into a known volume and measuring its vapor pressure at $\sim 40^{\circ} \mathrm{C}$ following the experimental procedure described by Scheidegger et al. [2010]. Gas purification and separation is performed in a new, smaller, and simplified (e.g., without a capillary) version of the water and noble gas extraction system described by Beyerle et al. [2000]. The new line is tailored to process the very small amounts of water and noble gases 
Table 1. Noble Gas Concentrations and Water Yields for Dimarshim Samples Using the CVCS System ${ }^{\mathrm{a}}$

\begin{tabular}{|c|c|c|c|c|c|c|c|c|c|c|c|}
\hline & $\begin{array}{l}\text { Sample } \\
\text { weight } \\
\text { [g] }\end{array}$ & $\begin{array}{l}\text { Water } \\
\text { yield } \\
\text { [g] } \\
\times 10^{-4}\end{array}$ & \pm & $\begin{array}{l}\mathrm{Ne} \\
{\left[\mathrm{cm}^{3} \mathbf{S T P} / \mathrm{g}\right]} \\
\times 10^{-6}\end{array}$ & \pm & $\begin{array}{l}\mathrm{Ar} \\
{\left[\mathrm{cm}^{3} \mathbf{S T P} / \mathrm{g}\right]} \\
\mathrm{x} 10^{-4}\end{array}$ & \pm & $\begin{array}{l}\mathbf{K r} \\
{\left[\mathbf{c m}^{3} \mathbf{S T P} / \mathbf{g}\right]} \\
\times 10^{-8}\end{array}$ & \pm & $\begin{array}{l}\mathrm{Xe} \\
{\left[\mathrm{cm}^{3} \mathbf{S T P} / \mathrm{g}\right]} \\
\mathrm{x} 10^{-8}\end{array}$ & \pm \\
\hline $\mathrm{ASW}_{(350 / 26)}$ & & & & 0.17 & & 2.67 & & 5.78 & & 0.77 & \\
\hline D1-c & 0.30 & 4.94 & 0.04 & $\begin{array}{l}3.15 \\
0.4\end{array}$ & 0.04 & n.a. & & n.a. & & n.a. & \\
\hline D1-m & 0.34 & 1.74 & 0.03 & $\begin{array}{l}1.47 \\
5\end{array}$ & 0.03 & $\begin{array}{l}8.43 \\
5\end{array}$ & 0.15 & $\begin{array}{l}12.38 \\
5\end{array}$ & 0.24 & $\begin{array}{l}1.32 \\
8\end{array}$ & 0.05 \\
\hline D1-f & 0.28 & 0.69 & 0.02 & $\begin{array}{l}0.42 \\
31\end{array}$ & 0.02 & $\begin{array}{l}3.04 \\
10\end{array}$ & 0.11 & $\begin{array}{l}5.53 \\
8\end{array}$ & 0.25 & $\begin{array}{l}0.77 \\
17\end{array}$ & 0.07 \\
\hline D1.1-c & 0.32 & 0.53 & 0.02 & $\begin{array}{l}2.43 \\
23\end{array}$ & 0.11 & $\begin{array}{l}14.54 \\
17\end{array}$ & 0.63 & $\begin{array}{l}20.76 \\
15\end{array}$ & 0.92 & $\begin{array}{l}2.06 \\
22\end{array}$ & 0.11 \\
\hline D1.1-m & 0.56 & 1.17 & 0.03 & $\begin{array}{l}1.07 \\
29\end{array}$ & 0.03 & $\begin{array}{l}7.32 \\
13\end{array}$ & 0.18 & $\begin{array}{l}11.26 \\
10\end{array}$ & 0.29 & $\begin{array}{l}1.25 \\
13\end{array}$ & 0.05 \\
\hline D1.2-c & 0.19 & 0.28 & 0.02 & $\begin{array}{l}2.52 \\
14\end{array}$ & 0.20 & $\begin{array}{l}18.16 \\
14\end{array}$ & 1.35 & $\begin{array}{l}27.42 \\
11\end{array}$ & 2.06 & $\begin{array}{l}2.73 \\
24\end{array}$ & 0.24 \\
\hline D1.2-m & 0.78 & 1.12 & 0.03 & $\begin{array}{l}1.15 \\
18\end{array}$ & 0.04 & $\begin{array}{l}9.16 \\
12\end{array}$ & 0.23 & $\begin{array}{l}14.00 \\
11\end{array}$ & 0.41 & $\begin{array}{l}1.39 \\
18\end{array}$ & 0.06 \\
\hline D1.2-f & 0.25 & 0.12 & 0.02 & $\begin{array}{l}1.37 \\
19\end{array}$ & 0.27 & $\begin{array}{l}9.94 \\
30\end{array}$ & 1.71 & $\begin{array}{l}16.71 \\
23\end{array}$ & 2.89 & $\begin{array}{l}1.64 \\
58\end{array}$ & 0.33 \\
\hline
\end{tabular}

${ }^{\mathrm{a} C}$ Coarse, medium, and fine grain size fractions are indicated by the suffixes $-\mathrm{c},-\mathrm{m}$, and $-\mathrm{f}$, respectively. Numbers in italics given beneath each noble gas concentration refer to the percentage the measured sample gas amounts are corrected for by the gas amounts released during the respective re-extractions. Uncertainties of noble gas concentrations are $1 \sigma$ and include raw data statistics, and uncertainties propagated from interference correction, calibration, and re-extraction correction. Also included are the uncertainties associated with the determination of the water amounts Vogel et al. [2013]. The error associated with gas calibration includes raw data statistics of the analyzed air aliquots and the error derived from the long term reproducibility of standard gas amounts, which are $1 \%$ for $\mathrm{He}$, Ne, Ar, and $\mathrm{Kr}$, respectively, and $2.5 \%$ for Xe. Noble gas concentrations of air-saturated water (ASW) are given for comparison and were calculated using the atmospheric pressure at the elevation of Dimarshim Cave of approx. $350 \mathrm{~m}$ a.s.l. and an average cave paleo-temperature of $\sim 26^{\circ} \mathrm{C}$.

released from stalagmite samples. After determination of the water mass, the noble gases are separated from the water by pulling the gas through a 3 $\AA$ molecular sieve at room temperature into a trap of activated charcoal held at $-196^{\circ} \mathrm{C}$. While the water is mostly trapped by the molecular sieve [cf., Beyerle et al., 2000], the charcoal separates the noncondensable He-Ne fraction from the condensable Ar-Kr-Xe fraction. The latter, together with residual water and other active gases, is quantitatively trapped at the charcoal. Prior to expansion of the He-Ne fraction to the spectrometer, it is further purified by admission to two more cryogenic traps $\left(-196^{\circ} \mathrm{C}\right.$ and $-203^{\circ} \mathrm{C}$, respectively) and a $\mathrm{Zr}-\mathrm{V}-\mathrm{Fe}$ getter pump (CapaciTorr ${ }^{\circledR} \mathrm{D}-400$ with a C400-2 DSK St172 cartridge from SAES ${ }^{\circledR}$ getters S.p.A.) operated at $\sim 150^{\circ} \mathrm{C}$. During the analysis of $\mathrm{He}$ and $\mathrm{Ne}$, the Ar-Kr-Xe fraction is released by heating the charcoal to $180^{\circ} \mathrm{C}$, and is subsequently purified by two $\mathrm{Zr}-\mathrm{V}-\mathrm{Fe}$ getter pumps (a ST707/ Pill getter and a CapaciTorr ${ }^{\circledR} \mathrm{D}$ 400 with a C400-2 DSK St172 cartridge, both from SAES ${ }^{\circledR}$ getters S.p.A.) operated at $\sim 350$ and $\sim 150^{\circ} \mathrm{C}$, respectively. The purified $\mathrm{Ar}-\mathrm{Kr}-\mathrm{Xe}$ fraction is expanded into the spectrometer after the $\mathrm{He}-\mathrm{Ne}$ fraction has been pumped off.

\subsection{Noble Gas Analysis}

[15] For paleotemperature determinations our focus is on accurate noble gas amounts rather than their isotopic compositions, which (with the exception of $\mathrm{He}$ ) have shown to be atmospheric in water [e.g., Brennwald et al., 2013; Kipfer et al., 2002]. Therefore, we analyze only one isotope of each stable noble gas $\left({ }^{4} \mathrm{He},{ }^{22} \mathrm{Ne},{ }^{86} \mathrm{Kr}\right.$, and $\left.{ }^{136} \mathrm{Xe}\right)$, except for $\mathrm{Ar}\left({ }^{36,40} \mathrm{Ar}\right)$. The $\mathrm{He}-\mathrm{Ne}$ and $\mathrm{Ar}-\mathrm{Kr}-\mathrm{Xe}$ fractions are sequentially analyzed using a noncommercial $90^{\circ}$ sector field noble gas mass spectrometer equipped with a highly linear, i.e., sample-size independent sensitivity, Baur-Signer source [Baur, 1980; see, also Beyerle et al., 2000], a Faraday cup for the measurement of more intense ion beams $\left({ }^{4} \mathrm{He},{ }^{36,40} \mathrm{Ar}\right)$, and a channeltron electron multiplier for ion counting weaker ones $\left({ }^{22} \mathrm{Ne},{ }^{86} \mathrm{Kr},{ }^{136} \mathrm{Xe}\right)$. The spectrometer is operated in peak jumping mode and at an electron energy of $100 \mathrm{eV}$. At this energy and with a mass resolution of the spectrometer $\mathrm{m} / \Delta \mathrm{m}$ of $\sim 70$, an interference correction on mass 22 from doubly charged $\mathrm{CO}_{2}$ is required. The ratio $\left(\mathrm{CO}_{2}\right)^{++} /\left(\mathrm{CO}_{2}\right)^{+}$, which is regularly checked analyzing the noble gas background in the "empty" spectrometer, averages at 
$(7.3 \pm 0.2) \times 10^{-3}$ and is very stable. The correction on mass 22 can amount to up to several \%, hence, $\mathrm{CO}_{2}$ is monitored throughout the analysis of the He-Ne fraction.

[16] To calibrate the system, an aliquot of a diluted air standard is measured before each sample. The noble gas amounts in these air aliquots do not deviate by more than 1-2 orders of magnitude from those released from a typical sample. The calibration gas is processed equivalently to the sample gas. Reciprocal sensitivities are $4.5 \times 10^{4}$ and $4.2 \times 10^{3} \mathrm{~cm}^{3}$ STP/A for ${ }^{4} \mathrm{He}$ and ${ }^{40} \mathrm{Ar}$, and $3.9 \times 10^{-15}, 4.3 \times 10^{-16}$, and $3.4 \times 10^{-16} \mathrm{~cm}^{3}$ $\mathrm{STP} / \mathrm{Hz}$ for ${ }^{22} \mathrm{Ne},{ }^{86} \mathrm{Kr}$, and ${ }^{136} \mathrm{Xe}$, respectively. Variations of the spectrometer sensitivities are monitored by daily measurements of known amounts of mixtures of $\mathrm{He}-\mathrm{Ne}$ and $\mathrm{Ar}-\mathrm{Kr}-\mathrm{Xe}$, respectively, that are directly admitted to the spectrometer [cf., Beyerle et al., 2000]. These variations are $\leq 1 \%$ for all analyzed noble gas isotopes for the duration of a run $(\sim 1$ week, including the analyses of the three grain size fractions of one sample and associated blanks and re-extractions). Uncertainties of the noble gas concentrations in Table 1 are presented at the $1 \sigma$ level and include raw data statistics, and uncertainties propagated from interference correction, calibration, and blank correction. Also included are the uncertainties associated with the determination of the water amounts. See caption of Table 1 for more information.

\subsection{Procedural Blanks and Re- Extractions}

[17] Together with each sample, two types of "blank" measurements are carried out routinely. Prior to every sample extraction a so-called procedural blank is analyzed, which simulates a full sample gas extraction, but without heating the respective sample. Procedural blanks provide information about noble gas backgrounds in the system. After the subsequent sample extraction, a re-extraction step is performed using the same extraction parameters as for the sample extraction. The re-extraction is used to estimate the combined noble gas amounts that (i) contribute to the blank of the sample extraction step at elevated temperatures and (ii) are released from the sample but are mostly not associated with inclusion water (see section 3.3.2).

\subsubsection{Procedural Blanks}

[18] Procedural blank levels in $\mathrm{cm}^{3} \mathrm{STP}$ scatter around $2 \times 10^{-10}(\mathrm{He}), 4 \times 10^{-12}(\mathrm{Ne}), 4 \times 10^{-9}$
(Ar), $3 \times 10^{-13}(\mathrm{Kr})$, and $2 \times 10^{-13}(\mathrm{Xe})$. Their $\mathrm{Ne}, \mathrm{Kr}$, and $\mathrm{Xe}$ elemental compositions are consistent with an adsorbed atmospheric signature (Figure 2b, see figure caption for an explanation of "adsorbed atmospheric composition"), reflecting the fact that light noble gases adhere much less tightly to recipient walls and sample surfaces than heavier ones. The elevated amounts of $\mathrm{He}$ in the procedural blanks (expressed, e.g., in high $\mathrm{He} / \mathrm{Kr}$ ratios in Figure 2a) are attributed to radiogenic $\mathrm{He}$ emitted from the glass fingers even at room temperature (discussed in detail in the next section). Also $\mathrm{Ar}$ is elevated in our line (Figure 2c) due to small contributions from the new $\mathrm{Zr}-\mathrm{V}-\mathrm{Fe}$ pill getters used for gas purification. Helium and Ar levels in the procedural blanks have been decreasing continuously during the runs and are expected to decrease further.

\subsubsection{Re-Extractions}

[19] Noble gas amounts released during re-extraction steps are (in $\mathrm{cm}^{3} \mathrm{STP}$ and corrected for procedural blank contributions) in the range of $2 \times$ $10^{-9}(\mathrm{He}), 2 \times 10^{-11}(\mathrm{Ne}), 7 \times 10^{-9}(\mathrm{Ar}), 8 \times$ $10^{-13}(\mathrm{Kr})$, and $1 \times 10^{-13}(\mathrm{Xe})$. Helium amounts in the re-extraction steps are very similar to those released during the main sample extraction steps, and also to those released during heating an empty glass finger (data not shown). This clearly indicates that ${ }^{4} \mathrm{He}$ released during the sample extraction steps originates predominantly from the glass fingers (glass 8250, Schott ${ }^{\circledR}$ ), which contain traces of $\mathrm{U}$ and $\mathrm{Th}$. Helium can therefore not be used for temperature determination, and is excluded from any further discussion. In contrast, $\mathrm{Ne}$ to $\mathrm{Xe}$ amounts in the re-extraction steps are lower by factors of $\sim 5(\mathrm{Ne}), \sim 7(\mathrm{Ar}), \sim 8(\mathrm{Kr})$, and $\sim 4(\mathrm{Xe})$ compared to average sample gas amounts, and are elementally broadly consistent with an atmospheric composition (Figures $2 \mathrm{~b}$ and $2 \mathrm{c}$ ). Hence, re-extraction noble gases are largely attributed to air released from small air inclusions within calcite crystals that had survived the crushing procedure. For Ar, Kr, and Xe, a very small contribution of a water-related noble gas component [air-saturated water (ASW); see also caption of Figures 2 and 3] is detectable as well, because re-extraction data points in Figure 2c plot on average slightly off the air end-member toward ASW. This is in line with the observation that during some reextractions very small amounts of water (e.g., $\leq 0.0045 \mathrm{mg}$, corresponding to $\leq 4 \%$ compared to the water amounts released during extraction of the medium samples) were released. The amounts of noble gases that could be dissolved in 
such small an amount of water (calculated according to the temperature and pressure conditions in Dimarshim Cave) contribute at most $\sim 20 \%$ to the total amounts of $\mathrm{Ar}, \mathrm{Kr}$, and $\mathrm{Xe}$ released during the re-extractions, i.e., most of the noble gases released in a re-extraction step are not related to inclusion water. Additionally, we find in the reextraction steps a small enrichment of Ne relative to the elemental composition of air (Figure 2b). A similar "extra Ne" component has already been described earlier [Ayliffe et al., 1993; Kluge et al., 2008; Scheidegger et al., 2010] and was attributed to $\mathrm{Ne}$ that is trapped within the calcite lattice of the stalagmite and is released diffusively from the sample at elevated temperatures [Scheidegger et al., 2010].
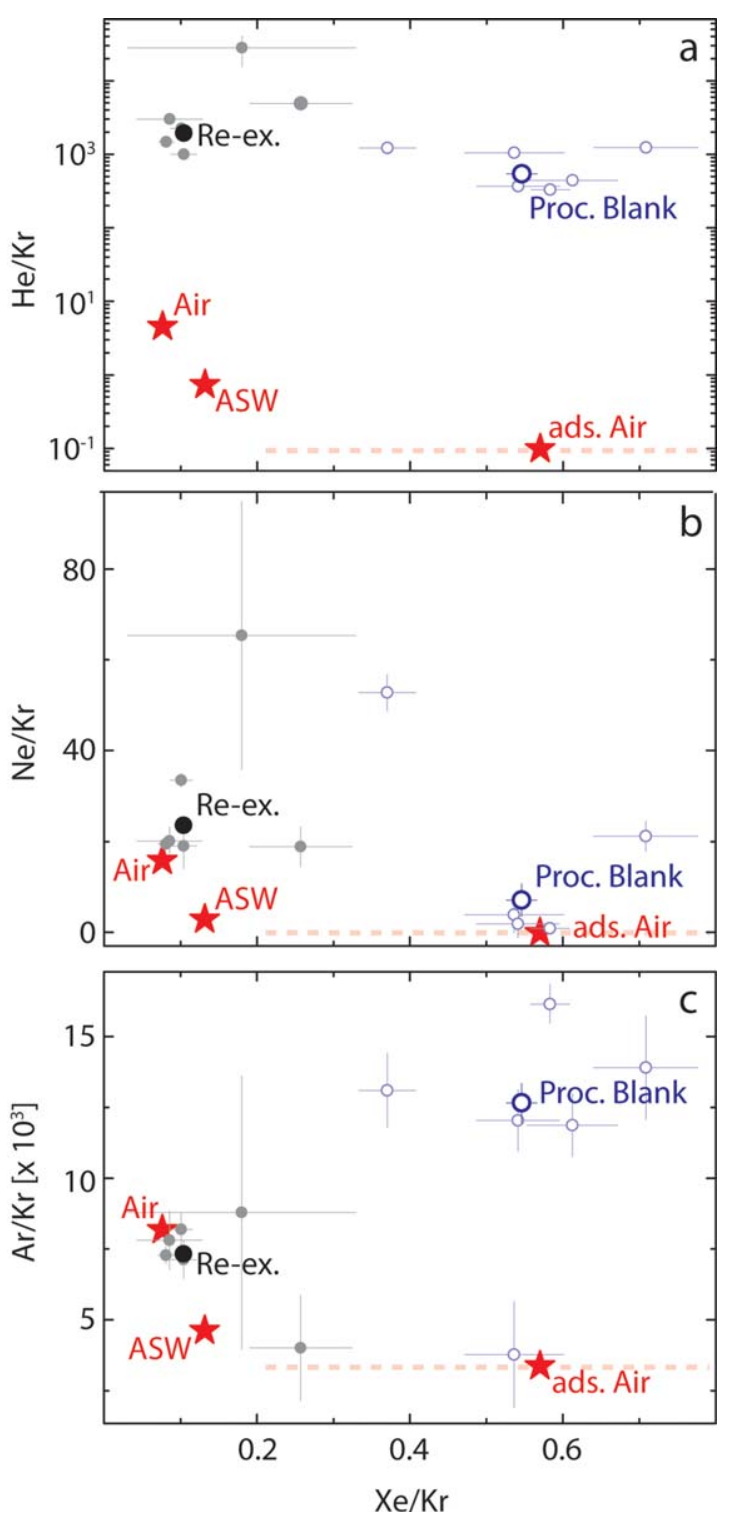

[20] It is obvious that a release of atmospheric noble gases from air inclusions and a diffusive release of $\mathrm{Ne}$ as observed in the re-extractions also take place during the main sample extraction steps. Therefore, it is possible, at least to a certain degree, to correct for these noble gas components by subtracting the gas amounts released during the reextraction steps from those measured in the respective sample extraction steps. This correction is performed here, using the re-extraction gas amounts uncorrected for procedural blank levels. The correction is to be considered a "minimum correction", as larger fractions of the gas components "inclusion air" and "lattice trapped Ne" are more likely to be released during the first heating step, i.e., during sample extraction, than during the subsequent re-extraction step. This is of minor consequence for $\mathrm{Ar}, \mathrm{Kr}$, and $\mathrm{Xe}$, because the algorithm used for temperature determination anyway deconvolves the sample gas in terms of the two end-member compositions air and ASW (cf., section 5.3). However, the lattice trapped $\mathrm{Ne}$ is not corrected for by the algorithm, and an insufficient correction of this component via the re-extraction can hamper NGT determination, as shown in section 5.3.

[21] The corrections of the sample gas amounts for re-extraction contributions are given in percentage of the measured sample gas amounts for each individual sample in Table 1. For the medium grain size fractions these contributions are around 18\% $(\mathrm{Ne}), 10 \%(\mathrm{Ar}), 8 \%(\mathrm{Kr})$, and $13 \%(\mathrm{Xe})$, and are on average $\leq 3 \%$ for the associated water masses.

Figure 2. Elemental compositions (a) $\mathrm{He} / \mathrm{Kr}$ versus $\mathrm{Xe} / \mathrm{Kr}$, (b) $\mathrm{Ne} / \mathrm{Kr}$ versus $\mathrm{Xe} / \mathrm{Kr}$, and (c) $\mathrm{Ar} / \mathrm{Kr}$ versus $\mathrm{Xe} / \mathrm{Kr}$ of procedural blanks and re-extractions. Small light blue open circles: individual procedural blanks; Large dark blue open circle: error weighted average procedural blank. Small grey solid circles: individual re-extractions; Large black solid circle: error weighted average re-extraction. For these plots, reextractions have been corrected for procedural blank contributions. Also shown are the elemental compositions of air, ASW, and adsorbed air (red star symbols). ASW elemental ratios are calculated for Dimarshim Cave temperature and pressure conditions using the solubility data recommended by Kipfer et al. [2002]. The dashed light red line reflects the Xe/ $\mathrm{Kr}$ compositional range of adsorbed air, characterized by a considerable enrichment of Xe relative to $\mathrm{Kr}$ and atmospheric composition. The used compositional range of adsorbed air $(\mathrm{Ar} / \mathrm{Kr}: 3400 \pm 850 ; \mathrm{Xe} / \mathrm{Kr}: 0.57 \pm 0.36)$ is based on noble gases adsorbed onto shales [Ozima and Podosek, 2002] and the noble gas compositions of meteorites that had undergone substantial terrestrial weathering [Scherer et al., 1994]. Adsorbed atmospheric $\mathrm{He} / \mathrm{Kr}$ and $\mathrm{Ne} / \mathrm{Kr}$ ratios are assumed to be close to zero. 


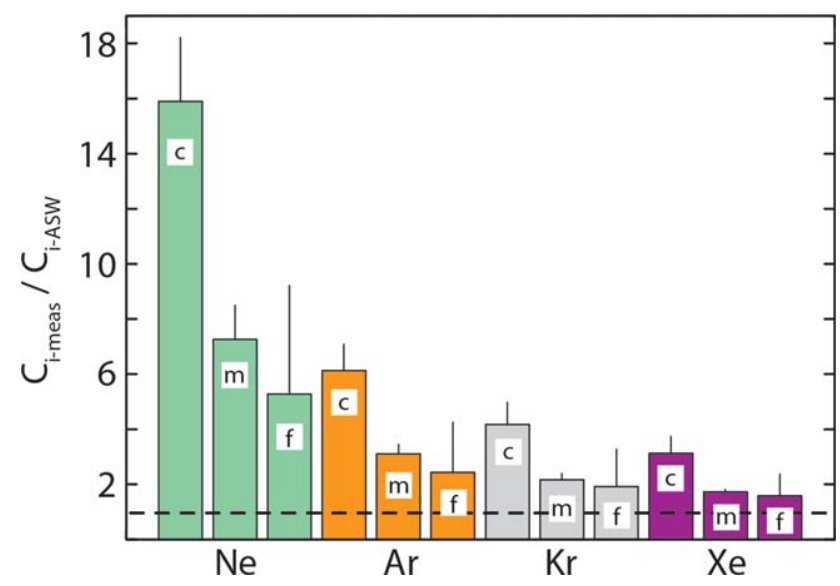

Figure 3. Average excesses of measured noble gas concentrations $\left(\mathrm{C}_{\mathrm{i} \text {-meas }} ; i=\mathrm{Ne}, \mathrm{Ar}, \mathrm{Kr}, \mathrm{Xe}\right)$ over noble gas concentrations in air-saturated water $\left(\mathrm{C}_{\mathrm{i}-\mathrm{ASW}}\right)$ for coarse $(\mathrm{c})$, medium $(\mathrm{m})$, and fine (f) grain size fractions, respectively. Error bars reflect standard deviations of average excesses. The dashed line represents ASW noble gas concentrations calculated for Dimarshim Cave temperature and pressure conditions using the solubility data recommended by Kipfer et al. [2002].

\section{Results and Discussion}

\subsection{Sample Gas Concentrations}

[22] $\mathrm{Ne}, \mathrm{Ar}, \mathrm{Kr}$, and $\mathrm{Xe}$ amounts released during sample extractions (corrected for re-extraction contributions) commonly exceed those expected for pure ASW noble gases from the respective amounts of concurrently extracted water (Figure 3 ). The observed noble gas excesses (defined as $\mathrm{C}_{\mathrm{i} \text {-meas }} / \mathrm{C}_{\mathrm{i}-\mathrm{ASW}}$, see caption of Figure 3 ) are attributed to air released during sample extraction from air-filled inclusions, which had not been opened during sample crushing. The excesses above ASW levels are highest for $\mathrm{Ne}$ and decrease toward Xe (Figure 3), reflecting the increasing solubility of noble gases in water with increasing atomic mass [e.g., Kipfer et al., 2002]. The Ne-Xe excesses are substantially lower in the medium and fine grain size fractions compared to those in the coarse fractions. In comparison to total extractions of D1 samples [Scheidegger et al., 2010; samples labelled "A"] $\mathrm{C}_{\mathrm{i} \text {-meas }} / \mathrm{C}_{\mathrm{i}-\mathrm{ASW}}$ ratios in the grain separates produced with the CVCS technique are reduced by up to 2 orders of magnitude. Therefore, a sample, originally inappropriate for NGT determination due to its high air content, might well become suitable for NGT determination if processed with the CVCS system.

\subsection{Element Ratios}

[23] In both plots in Figure 4, the Dimarshim data points fall within the $95 \%$ confidence level on or close to the mixing lines between the end-member compositions of air and ASW, indicating that the sample gases are binary mixtures of air- and ASW-related noble gases without sizeable admixtures of further components, such as adsorbed air. This clearly demonstrates that, in contrast to the previous offline crushing technique [Scheidegger et al., 2010; Scheidegger et al., 2011], the CVCS system allows crushing samples without adding (elementally fractionated) atmospheric noble gases that would hamper a later NGT determination [cf., Brennwald et al., 2013, Figure ].

\subsection{Paleotemperatures}

[24] As shown above, the CVCS technique allows to reduce the air content of a given sample without introducing additional noble gas components like elementally fractionated adsorbed air. For Ar, Kr, and $\mathrm{Xe}$, the extracted sample gases, therefore, largely represent binary mixtures of the two components air and ASW (for Ne see below), and both components are present in comparable proportions. These properties of the sample gas are prerequisites for a successful application of the MATLAB code "NOBLE90" [Aeschbach-Hertig et al., 1999] for NGT determination. The code deconvolves measured noble gas concentrations (e.g., Ar, $\mathrm{Kr}$, and $\mathrm{Xe}$ ) into the two end-member compositions "ASW" and "air" by error weighted least squares regression, and calculates an equilibration temperature from the ASW component using suitable noble gas solubility data [e.g., Kipfer et al., 2002]. The error weighted squared deviations between measured and modeled noble gas concentrations are minimized using a $\chi^{2}$ test. This provides a statistical assessment of whether a solution is well described by the assumption that measured noble gases 


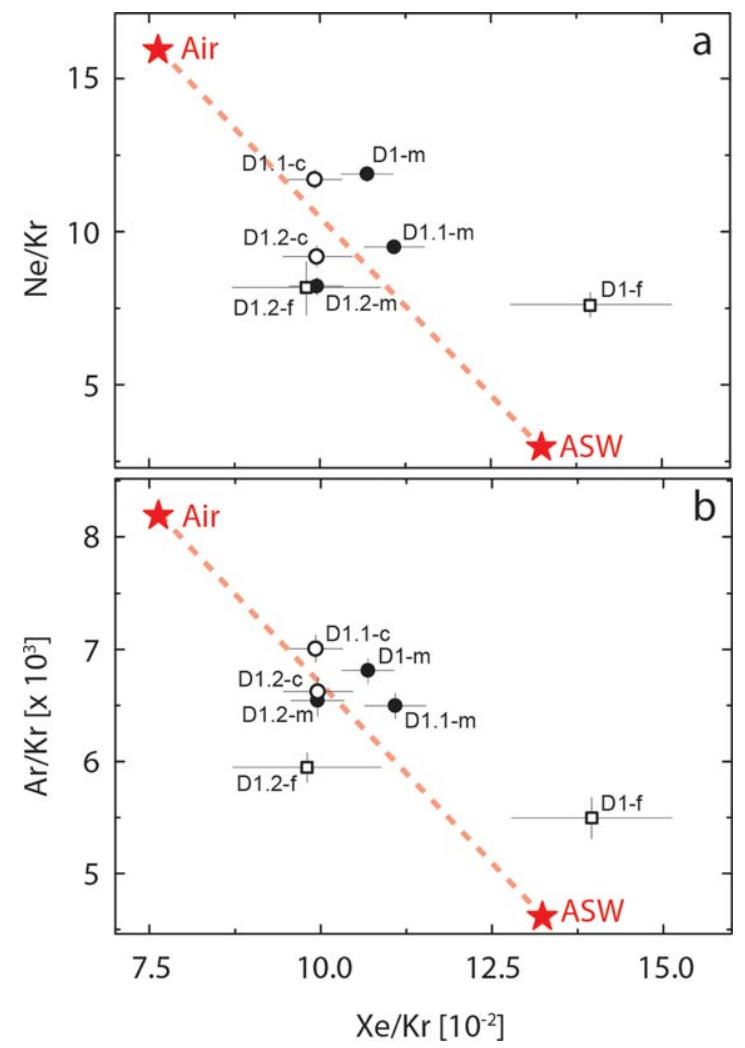

Figure 4. Elemental compositions (a) $\mathrm{Ne} / \mathrm{Kr}$ versus $\mathrm{Xe} / \mathrm{Kr}$ and (b) $\mathrm{Ar} / \mathrm{Kr}$ versus $\mathrm{Xe} / \mathrm{Kr}$ of coarse (open circles), medium (solid circles), and fine-grained (open squares) fractions of D1 samples in comparison to the end-member compositions of air and ASW. Error bars are $1 \sigma$ and represent the overall errors excluding the ones associated with the determination of water amounts. The dashed red lines represent the respective mixing lines between the two noble gas end-member compositions air and ASW. ASW noble gas concentrations are calculated for Dimarshim Cave temperature and pressure conditions and using the solubility data recommended by Kipfer et al. [2002]. We emphasize that NGTs cannot be calculated from the element ratios shown here, as their sensitivity to temperature is by far too small in the presence of even small amounts of air [cf., Kluge et al., 2008]. Instead they are calculated from the actual noble gas concentrations of the ASW component in each sample, as these are several times more sensitive to temperature variations than the associated element ratios.

represent a binary mixture from the reservoirs air and ASW. If so, the $\chi^{2}$ value should be close to the degrees of freedom of the model. For a more detailed description of the code and the statistical treatment of the data see, e.g., Aeschbach-Hertig et al. [1999, 2000] and Peeters et al. [2002], and the free online available manual for the program "NOBLE90" [Aeschbach-Hertig et al., 2003]. NGTs from fitting $\mathrm{Ar}, \mathrm{Kr}$, and $\mathrm{Xe}$ for each analyzed grain size fraction are summarized in the upper part of Table 2, and are visualized in Figure 5.
[25] The three medium grain size fractions (solid circles in figure 5) had been crushed closest to the "ideal" grain size as defined earlier, and, hence, had been expected to be most suitable for NGT determination. The resulting NGTs based on Ar, $\mathrm{Kr}$, and $\mathrm{Xe}$ concentrations are indeed, within $1 \sigma$ uncertainties, identical to each other and perfectly reproduce the paleotemperature of Dimarshim Cave. The $\chi^{2}$ values resulting from the calculation of these temperatures are well within the $95 \%$ confidence ranges permitted by the respective number of degrees of freedom (see Table 2) and, as a result, express the statistical agreement between the data and a binary-mixing model of air- and ASW-related noble gases. Also the NGTs derived from the two analyzed fine fractions (open squares) agree, within uncertainties, with the expected temperature range. However, the respective errors are large due to the considerable analytical uncertainties of the small noble gas and water amounts. This result indicates that crushing a sample to grains considerably smaller than the "ideal" grain size (see section 2) does not only effectively remove air from the samples, but also a large fraction of its inclusion water.

[26] NGTs derived from the two coarse fractions (open circles) are lower than expected, even taking into account the large uncertainties, which are attributed to the substantial corrections for airrelated noble gases. The low $\chi^{2}$ values associated with the temperatures derived from fine and coarse grain size fractions are, therefore, mainly a result of the large uncertainties of the input gas concentrations, and have only limited significance for judging the quality of the actual fits [cf., Aeschbach-Hertig et al., 1999].

[27] An alternative fitting procedure for our data set is the so-called ensemble fit [Peeters et al., 2002], as all of our samples have grown at the same temperature. For an ensemble fit NOBLE90 processes the noble gas concentrations of an entire set of samples simultaneously with the additional requirement that the resulting temperature must be the same for all samples. Thus, the ensemble fit has got a more favorable ratio of free to constrained parameters than an individual sample fit and might, therefore, provide more precise results. The ensemble fit of all samples (using $\mathrm{Ar}, \mathrm{Kr}$, and $\mathrm{Xe}$ for fitting) indeed results in a precise NGT of $26.4 \pm 1.7^{\circ} \mathrm{C}$ in excellent agreement with the paleotemperature of Dimarshim Cave (large violet star symbol in Figure 5; Table 2). The associated $\chi^{2}$ value of 17 is well within the $95 \%$ confidence range for 13 degrees of freedom and indicates that 
Table 2. NGTs for Dimarshim Samples Using the CVCS System ${ }^{\mathrm{a}}$

\begin{tabular}{|c|c|c|c|c|c|}
\hline & $\mathbf{T}\left[{ }^{\circ} \mathbf{C}\right]$ & $\pm($ scaled $)$ & $\pm(\operatorname{cov})$ & \#freedom & $\chi^{2}$ \\
\hline & \multicolumn{5}{|c|}{ Used for fitting: Ar, Kr, and Xe concentrations } \\
\hline$\overline{D 1-c}$ & n.d. & & & & \\
\hline D1-m & 27.1 & 4.2 & 2.7 & 1 & 2.4 \\
\hline D1-f & 32.5 & 5.4 & 3.7 & 1 & 2.1 \\
\hline D1.1-c & 17.2 & 1.4 & 5.0 & 1 & 0.1 \\
\hline D1.1-m & 24.6 & 2.9 & 2.8 & 1 & 1.0 \\
\hline D1.2-c & 6.8 & 1.4 & 6.2 & 1 & 0.1 \\
\hline $\mathrm{D} 1.2-\mathrm{m}$ & 24.2 & 1.2 & 3.3 & 1 & 0.1 \\
\hline D1.2-f & 15.8 & 4.7 & 13.4 & 1 & 0.1 \\
\hline D1ensemble fit & 26.4 & 1.7 & 1.5 & 13 & 17.0 \\
\hline \multirow[t]{2}{*}{ D1ensemble fit (only medium fractions) } & 25.6 & 1.5 & 1.7 & 5 & 4.1 \\
\hline & \multicolumn{5}{|c|}{ Used for fitting: $\mathrm{Ne}, \mathrm{Ar}, \mathrm{Kr}$, and $\mathrm{Xe}$ concentrations } \\
\hline$\overline{D 1-c}$ & n.d. & & & & \\
\hline D1-m & 35.0 & 7.1 & 2.6 & 2 & 14.9 \\
\hline D1-f & 43.6 & 8.4 & 3.4 & 2 & 12.1 \\
\hline D1.1-c & 18.1 & 1.1 & 4.0 & 2 & 0.2 \\
\hline D1.1-m & 25.4 & 1.6 & 2.0 & 2 & 1.2 \\
\hline D1.2-c & 2.3 & 3.3 & 3.7 & 2 & 1.6 \\
\hline $\mathrm{D} 1.2-\mathrm{m}$ & 16.8 & 4.7 & 1.7 & 2 & 15.3 \\
\hline D1.2-f & 13.1 & 3.0 & 8.8 & 2 & 0.2 \\
\hline D1ensemble fit & 29.3 & 3.0 & 1.1 & 20 & 139 \\
\hline D1ensemble fit (only medium fractions) & 26.3 & 3.6 & 1.2 & 8 & 69 \\
\hline
\end{tabular}

${ }^{a}$ NGTs determined from concentrations of $\mathrm{Ar}, \mathrm{Kr}$, and $\mathrm{Xe}$ (upper part of table) and $\mathrm{Ne}, \mathrm{Ar}, \mathrm{Kr}$, and $\mathrm{Xe}$ (lower part of table) of Dimarshim samples using the MATLAB code "NOBLE90" [Aeschbach-Hertig et al., 1999]. N.d. = Not determined. Two types of uncertainties $(1 \sigma)$ are given with the temperatures: \pm (scaled) reflects the uncertainty of $\mathrm{T}$, scaled with $\left(\chi^{2} / \nu\right)^{1 / 2}$ to account for goodness of fit; \pm (cov) reflects the uncertainty of $\mathrm{T}$ as derived from propagating the errors of the input parameters. For the discussion, and also in Figure 5, we always use the larger of both uncertainties. Also given are the numbers of degrees of freedom (\# freedom), defined as the difference between the number of measured (e.g., Ar, $\mathrm{Kr}$, and Xe concentrations) and unknown parameters (temperature and amount of air). $\chi^{2}$ represents the sum of the error weighted squared deviations between modeled and measured gas concentrations, i.e. is a measure for the goodness of the respective fit to a binary mixture of noble gases from air and ASW [Aeschbach-Hertig et al., 1999]. For more information, see, e.g., Aeschbach-Hertig et al. [1999] and the free online available Manual for the program "NOBLE90" [Aeschbach-Hertig et al., 2003]

the data set as a whole is in statistical agreement with the underlying model. An ensemble fit using only the medium fractions results in a NGT of $25.6 \pm 1.7^{\circ} \mathrm{C}$, remarkably close to the value obtained by the ensemble fit using all grain size fractions. Again, the corresponding $\chi^{2}$ value of 4.1 is perfect given the mean value of 5 .

[28] Including $\mathrm{Ne}$ into the fitting procedure introduces more scatter among the resulting NGTs (see lower panel of Table 2), and most of the determined NGTs derived by individual fitting are inconsistent with the paleotemperature range of Dimarshim Cave. Also the $\chi^{2}$ values associated with the NGTs of the medium fractions clearly indicate that the data including $\mathrm{Ne}$ are not satisfactorily explained by a binary mixture of air and ASW-related noble gases. This result is corroborated by the ensemble fits including all, and only the medium fractions, respectively. While the nominal NGTs agree within uncertainties with the cave paleotemperature, $\chi^{2}$ values predominantly lie outside their respective $95 \%$ confidence level ranges (Table 2, lower part). Supposedly, the re-

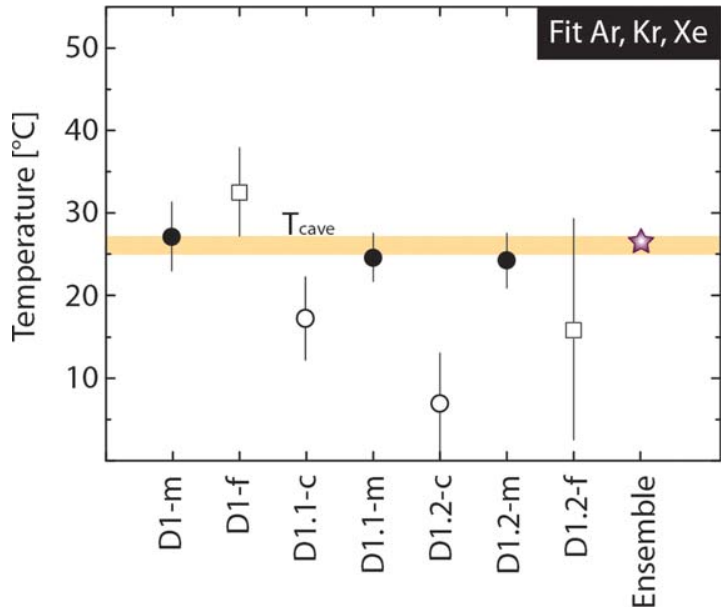

Figure 5. NGTs of Dimarshim samples using $\mathrm{Ar}, \mathrm{Kr}$, and $\mathrm{Xe}$ for fitting. Coarse fractions: open circles, medium fractions: solid circles; fine fractions: open squares; ensemble fit including all data points: violet star symbol. The orange area represents the paleotemperature of Dimarshim Cave $\left(T_{\text {cave }}\right.$; $25-27^{\circ} \mathrm{C}$ ). Error bars are $1 \sigma$ and represent the uncertainties of the temperatures taking into account all analytical uncertainties and also the quality of the fit between measured and modeled noble gas concentrations [Aeschbach-Hertig et al., 1999]. 
extraction correction did not quantitatively account for the amount of lattice trapped $\mathrm{Ne}$ released during sample extraction. We emphasize, however, that the re-extraction correction of the $\mathrm{Ar}, \mathrm{Kr}$, and $\mathrm{Xe}$ sample gas amounts is appropriate, not least because the determined NGTs, and especially those of the medium fractions and the ensemble fit, are robust and reproduce the expected paleo cave temperature accurately.

\section{Summary and Outlook}

[29] The new CVCS system allowed us to deduce robust temperature information from different samples of stalagmite D1 from Dimarshim Cave, Yemen. The same stalagmite had previously been used to test different water and noble gas extraction techniques [Scheidegger et al., 2010, 2011]. Using only $\mathrm{Kr}$ and Xe concentrations, Scheidegger et al. [2011] could estimate NGTs in agreement with the modern cave temperature for 7 out of 8 D1 samples, demonstrating that the noble gases dissolved in the inclusion water of stalagmite D1 indeed preserve a temperature signal. However, the authors could not include Ar in their data analysis for NGT determination, because Ar was substantially affected by an adsorbed atmospheric component. Therefore, a statistical assessment of the NGT results was not possible with only two measured parameters ( $\mathrm{Kr}$ and Xe concentrations) for a system with two unknowns (temperature, air content). In vacuo crushing with the CVCS system (i) decreases the air content of the samples, and (ii) avoids adsorption, and therefore allows to also use Ar for NGT determination. If a set of multiple samples grown at the same temperature is available, an ensemble fit can increase precision and reliability of the resulting NGT. For the D1 samples studied here, the uncertainty of the ensemble NGT is in the range of $1-2^{\circ} \mathrm{C}$. This precision is fully sufficient to identify temperature shifts, e.g., between glacial and interglacial periods often reaching temperature amplitudes of up to $10-12^{\circ} \mathrm{C}$ [e.g., Petit et al., 1999]. The ensemble fit result also indicates that, once the most suitable grain size fraction of a given sample has been found by analyzing all three grain size fractions, it should be sufficient to crush subsequent samples (from the same stalagmite) such that most of the material is concentrated in the optimum grain size fraction and only one instead of three analyses is required.

[30] Neon extracted from the samples is a mixture of noble gases from the three components air,
ASW, and probably lattice trapped Ne [Scheidegger et al., 2010]. If the latter component is too prominent, as it seems to be the case in the Dimarshim samples, Ne cannot be used for NGT determination. Therefore, in a next step we will test whether in vacuo milling of a grain separate at room temperature within its respective glass finger is capable of efficiently extracting water and noble gases without the concurrent release of lattice trapped Ne. If successful, also Ne would become available for routine NGT determination.

[31] In conclusion, the new CVCS system enables routine determinations of paleotemperatures from stalagmite samples with total water contents ideally at least in the range of $10^{-3} \mathrm{~g}$ of water per $\mathrm{g}$ of stalagmite rock, as it is the case for stalagmite D1 [Vogel et al., 2013]. In combination with precise stalagmite growth dating, the NGTs allow reconstructing past regional temperature evolutions. Such paleotemperature records are not only of paramount importance for temperature predictions, but will also help interpreting the often complex stable isotope records preserved in the stalagmites' calcite, for instance by disentangling temperature from other, e.g., hydrological signals.

\section{Acknowledgments}

[32] The authors would like to thank U. Menet and H. Baur for their assistance in the noble gas laboratory at ETH Zurich. Inspiring discussions with Y. Krüger (University of Berne) about the formation of fluid inclusions are greatly acknowledged. We also thank the associate editor Cin-Ty Lee for handling this manuscript and Sujoy Mukhopadhyay as well as one anonymous reviewer for their helpful and constructive reviews. This study was carried out in the framework of the Sinergia project "STALCLIM" funded by the Swiss National Science Foundation (grant no. CRSI22-132646/1 to D. Fleitmann and co-PI's).

\section{References}

Aeschbach-Hertig, W., F. Peeters, U. Beyerle, and R. Kipfer (1999), Interpretation of dissolved atmospheric noble gases in natural waters, Water Resour. Res., 35, 2779-2792.

Aeschbach-Hertig, W., F. Peeters, U. Beyerle, and R. Kipfer (2000), Palaeotemperature reconstruction from noble gases in groundwater accounting for equilibration with entrapped air, Nature, 405, 1040-1044.

Aeschbach-Hertig, W., F. Peeters, U. Beyerle, and R. Kipfer (2003), Manual for the program "NOBLE90". [Available at http://www.iup.uni-heidelberg.de/institut/forschung/ groups/aquasys/WAH/downloads/Noble90\%20Manual.pdf, accessed 14 May 2013.].

Affek, H. P., M. Bar-Matthews, A. Ayalon, A. Matthews, and J. M. Eiler (2008), Glacial/interglacial temperature 
variations in Soreq cave speleothems as recorded by "clumped isotope" thermometry, Geochim. Cosmochim. Acta, 72, 5351-5360.

Ayliffe, L. K., G. Turner, and P. G. Burnard (1993), Noble gas contents of speleothem inclusion fluids: Potential as indicators of precipitation temperature, Terra Nova, 5, 646.

Baur, H. (1980), Numerische simulation und praktische erprobung einer rotationssymmetrischen ionenquelle for gasmassenspektrometer, $\mathrm{PhD}$ thesis, ETH Zürich.

Beyerle, U., W. Aeschbach-Hertig, D. M. Imboden, H. Baur, T. Graf, and R. Kipfer (2000), A mass spectrometric system for the analysis of noble gases and tritium from water samples, Environ. Sci. Technol., 34, 2042-2050.

Brennwald, M. S., N. Vogel, Y. Scheidegger, Y. Tomonaga, D. M. Livingstone, and R. Kipfer (2013), Noble gases as environmental tracers in sediment porewaters and in stalagmite fluid inclusions, in The Noble Gases as Geochemical Tracers, edited by P. Burnard, p. 618, Springer, Berlin Heidelberg.

Doose-Rolinski, H., U. Rogalla, G. Scheeder, A. Lückge, and U. von Rad (2001), High-resolution temperature and evaporation changes during the late Holocene in the northeastern Arabian Sea, Paleoceanography, 16, 358-367.

Fairchild, I. J., and A. Baker (2012), Speleothem Science: From Process to Past Environments, 432 pp., Wiley-Blackwell, West Sussex, U. K.

Fairchild, I. J., C. L. Smith, A. Baker, L. Fuller, C. Spötl, D. Mattey, F. McDermott, and E.M.I.F. (2006), Modification and preservation of environmental signals in speleothems, Earth Sci. Rev., 75, 105-153.

Fleitmann, D., et al. (2007), Holocene ITCZ and Indian monsoon dynamics recorded in stalagmites from Oman and Yemen (Socotra), Quat. Sci. Rev., 26, 170-188.

Hendy, C. H., and A. T. Wilson (1968), Paleoclimatic data from speleothems, Nature, 219, 48-51.

Huguet, C., J.-H. Kim, J. S. S. Damsté, and S. Schouten (2006), Reconstruction of sea surface temperature variations in the Arabian Sea over the last $23 \mathrm{kyr}$ using organic proxies (TEX86 and U37K'), Paleoceanography, 21, PA3003, doi:10.1029/2005PA001215.

Kipfer, R., W. Aeschbach-Hertig, F. Peeters, and M. Stute (2002), Noble gases in lakes and ground waters, in Noble Gases in Geochemistry and Cosmochemistry, edited by D. Porcelli, C. J. Ballentine, and R. Wieler, pp. 615-700, The Mineral. Soc. of Am., Washington, D. C.
Kluge, T., T. Marx, D. Scholz, S. Niggemann, A. Mangini, and W. Aeschbach-Hertig (2008), A new tool for paleoclimate reconstruction: Noble gas temperatures from fluid inclusions in speleothems, Earth Planet. Sci. Lett., 269, 408-415.

Krüger, Y., D. Marti, R. H. Staub, D. Fleitmann, and M. Frenz (2011), Liquid-vapour homogenisation of fluid inclusions in stalagmites: Evaluation of a new thermometer for palaeoclimate research, Chem. Geol., 289, 39-47.

Ozima, M., and F. A. Podosek (2002), Noble Gas Geochemistry, 2nd ed., Cambridge Univ. Press, Cambridge, U. K.

Peeters, F., U. Beyerle, W. Aeschbach-Hertig, J. Holocher, M. S. Brennwald, and R. Kipfer (2002), Improving the noble gas based paleoclimate reconstruction and groundwater dating using $20 \mathrm{Ne} / 22 \mathrm{Ne}$ ratios, Geochim. Cosmochim. Acta, $67,587-600$.

Petit, J. R., et al. (1999), Climate and atmospheric history of the past 420,000 years from the Vostok ice core, Antarctica, Nature, 399, 429-435.

Scheidegger, Y. M., H. Baur, M. S. Brennwald, D. Fleitmann, R. Wieler, and R. Kipfer (2010), Accurate analysis of noble gas concentrations in small water samples and its application to fluid inclusions in stalagmites, Chem. Geol., 272, 31-39.

Scheidegger, Y. M., M. S. Brennwald, D. Fleitmann, P.-Y. Jeannin, R. Wieler, and R. Kipfer (2011), Determination of Holocene cave temperatures from $\mathrm{Kr}$ and $\mathrm{Xe}$ concentrations in stalagmite fluid inclusions, Chem. Geol., 288, 61-66.

Scherer, P., L. Schultz, and T. Loeken (1994), Weathering and atmopsheric noble gases in chondrites, in Noble Gas Geochemistry and Cosmochemistry, edited by J. Matsuda, pp. 43-53, Terra Sci., Tokyo.

Thompson, P., H. P. Schwarcz, and D. C. Ford (1974), Continental pleistocene climatic variations from speleothem age and isotopic data, Science, 184, 893-895.

Vogel, N., Y. Scheidegger, M. S. Brennwald, D. Fleitmann, S. Figura, R. Wieler, and R. Kipfer (2013), Stalagmite water content as a proxy for drip water supply in tropical and subtropical areas, Clim. Past, 8, 1-9.

Zhang, R., H. P. Schwarcz, D. C. Ford, F. S. Schroeder, and P. A. Beddows (2008), An absolute paleotemperature record from 10 to $6 \mathrm{ka}$ inferred from fluid inclusion $\mathrm{D} / \mathrm{H}$ ratios of a stalagmite from Vancouver Island, British Columbia, Canada, Geochim. Cosmochim. Acta, 72, 1014-1026. 